\title{
Modern space-time and undecidability
}

\author{
Rodolfo Gambini \\ Instituto de Física, Facultad de Ciencias, Universidad de la República, Iguá 4225, CP 11400 Montevideo, Uruguay \\ Jorge Pullin \\ Department of Physics and Astronomy, Louisiana State University, Baton Rouge, LA 70803-4001
}

(Dated: December 5th. 2007)

\begin{abstract}
The picture of space-time that Minkowski created in 1907 has been followed by two important developments in physics not contained in the original picture: general relativity and quantum mechanics. We will argue that the use of concepts of those theories to construct space-time implies conceptual modifications in quantum mechanics. In particular one can construct a viable picture of quantum mechanics without a reduction process that has outcomes equivalent to a picture with a reduction process. One therefore has two theories that are entirely equivalent experimentally but profoundly different in the description of reality they give. This introduces a fundamental level of undecidability in physics of a kind that has not been present before. We discuss some of the implications.
\end{abstract}

\section{INTRODUCTION}

In 1907 Minkowski noted that the natural arena to formulate the special theory of relativity of Einstein was spacetime. In his address delivered at the 80th Assembly of German Natural Scientists and Physicians in 1908, Minkowski remarks: "The views of space and time which I wish to lay before you have sprung from the soil of experimental physics, and therein lies their strength. They are radical. Henceforth space by itself, and time by itself, are doomed to fade away into mere shadows, and only a kind of union of the two will preserve an independent reality.". "The soil of experimental physics" in 1907 differs from the one today. At that time it was thought that space and time were continuous and that they could be measured with arbitrary precision. This was altered when quantum mechanics was developed in the 1930's. There it was noted that fundamental uncertainties were germane to modern physics. In 1957 Salecker and Wigner [1] decided to revise the picture that Minkowski had painted of space-time introducing concepts of quantum mechanics. In particular they considered limitations in the accuracy of clocks and rulers that one needs in order to construct the picture of space-time that we are familiar with. But fundamental uncertainties in quantum mechanics can be minimized. The situation changes dramatically when general relativity is brought into the picture in two different ways. On the one hand general relativity leads to fundamental uncertainties that cannot be minimized. In particular one cannot measure distances and times beyond a minimum level of uncertainty, as was emphasized by Ng and Van Dam [2]. The second way in which general relativity alters the picture is that space-time is now a dynamical arena that is not directly observable. The only observable properties of space-time are relational in nature. Therefore unlike the space-time of Minkowski, which was an immutable arena for special relativity, in general relativity space-time becomes an object that cannot be directly probed and the properties of it that can be probed are relational in nature and subject to fundamental minimum uncertainties.

To further elaborate the point, consider the measurement of time. In its usual formulation, quantum mechanics involves an idealization. The idealization is the use of a perfect classical clock to measure times. Such a device clearly does not exist in nature, since all measuring devices are subject to some level of quantum fluctuations. Therefore the equations of quantum mechanics, when cast in terms of the variable that is really measured by a clock in the laboratory, will differ from the traditional Schrödinger description. Although this is an idea that arises naturally in ordinary quantum mechanics, it is of paramount importance when one is discussing quantum gravity. This is due to the fact that general relativity is a generally covariant theory where one needs to describe the evolution in a relational way. One ends up describing how certain objects change when other objects, taken as clocks, change. At the quantum level this relational description will compare the outcomes of measurements of quantum objects. Quantum gravity is expected to be of importance in regimes (e.g. near the big bang or a black hole singularity) in which the assumption of the presence of a classical clock is clearly unrealistic. The question therefore arises: is the difference between the idealized version of quantum mechanics and the real one just of interest in situations when quantum gravity is predominant, or does it have implications in other settings?

We will argue that indeed it does have wider implications. Some of them are relevant to conceptual questions (e.g. the black hole information paradox or ultimate limitations on quantum computing) and there might even be experimental implications. A detailed discussion of these ideas can be found in previous papers [3, 4, 5], and in particular in the pedagogical review [6]. Here we present an abbreviated discussion as an introduction to a remarkable consequence of these ideas: that the nature of physical processes in modern physics becomes undecidable. In a nutshell 
we observe that when one considers fundamental limitations in the measurements of space-time, unitary quantum mechanics behaves in the same way as quantum mechanics with a reduction process. The two theories therefore become equivalent in their physical predictions, yet they are radically different in nature: in one of them quantum states are determined once and for all at the beginning of the universe and are not changed by physical events. In the other the picture is dramatically different, with quantum states constantly changing as events produces measurable outcomes. In spite of these differences, there is no experimental way to decide which of these pictures corresponds to reality. The implications of this observation philosophically are profound. They advocate what is technically known in philosophical circles as a "regularist" point of view towards the nature of physical laws, drastically different from the "necessitarian" point of view that was considered at the time of Minkowski's conception of space-time as the most natural interpretation of the role of physical laws.

The plan of this paper is as follows: in the next section we will discuss the form of the evolution equation of quantum mechanics when the time variable, used to describe it, is measured by a real clock. In section III we will consider a fundamental bound on how accurate can a real clock be and the implications it has for quantum mechanics in terms of real clocks and its consequences. Section IV discusses briefly some possible experimental implications of the proposal. Section V discusses conceptual implications in the foundations of quantum mechanics. Section VI argues that the conceptual implications leads to a new notion of undecidability in the nature of physical processes in quantum theory. We end with a discussion.

\section{QUANTUM MECHANICS WITH REAL CLOCKS}

Given a physical situation we start by choosing a "clock". By this we mean a physical quantity (more precisely a set of quantities, like when one chooses a clock and a calendar to monitor periods of more than a day) that we will use to keep track of the passage of time. An example of such a variable could be the angular position of the hand of an analog watch. Let us denote it by $T$. We then identify some physical variables that we wish to study as a function of time. We shall call them generically $O$ ("observables"). We then proceed to quantize the system by promoting all the observables and the clock variable to self-adjoint quantum operators acting on a Hilbert space. The latter is defined once a well defined inner product is chosen in the set of all physically allowed states. Usually it consists of squared integrable functions $\psi(q)$ with $q$ the configuration variables.

Notice that we are not in any way modifying quantum mechanics. We assume that the system has an evolution in terms of an external parameter $t$, which is a classical variable, given by a Hamiltonian and with operators evolving with Heisenberg's equations (it is easier to present things in the Heisenberg picture, though it is not mandatory to use it for our construction). Then the standard rules of quantum mechanics and its probabilistic nature apply.

We will call the eigenvalues of the "clock" operator $T$ and the eigenvalues of the "observables" $O$. We will assume that the clock and the measured system do not interact (if one considered an interaction it would produce additional effects to the one discussed). So the density matrix of the total system is a direct product of that of the system under study and the clock $\rho=\rho_{\mathrm{sys}} \otimes \rho_{\mathrm{cl}}$, and the system evolves through a unitary evolution operator that is of the tensor product form $U=U_{\text {sys }} \otimes U_{\text {cl }}$. The quantum states are described by a density matrices at a time $t$. Since the latter is unobservable, we would like to shift to a description where we have density matrices as functions of the observable time $T$. We define the probability that the resulting measurement of the clock variable $T$ correspond to the value $t$,

$$
\mathcal{P}_{t}(T) \equiv \frac{\operatorname{Tr}\left(P_{T}(0) U_{\mathrm{cl}}(t) \rho_{\mathrm{cl}} U_{\mathrm{cl}}(t)^{\dagger}\right)}{\int_{-\infty}^{\infty} d t \operatorname{Tr}\left(P_{T}(t) \rho_{\mathrm{cl}}\right)}
$$

where $P_{T}(0)$ is the projector on the eigenspace with eigenvalue $T$ evaluated at $t=0$. We note that $\int_{-\infty}^{\infty} d t \mathcal{P}_{t}(T)=1$. We now define the evolution of the density matrix,

$$
\rho(T) \equiv \int_{-\infty}^{\infty} d t U_{\mathrm{sys}}(t) \rho_{\mathrm{sys}} U_{\mathrm{sys}}(t)^{\dagger} \mathcal{P}_{t}(T)
$$

where we dropped the "sys" subscript in the left hand side since it is obvious we are ultimately interested in the density matrix of the system under study, not that of the clock.

We have therefore ended with an "effective" density matrix in the Schrödinger picture given by $\rho(T)$. It is possible to reconstruct entirely in a relational picture the probabilities using this effective density matrix, for details we refer the reader to the lengthier discussion in [6]. By its very definition, it is immediate to see that in the resulting evolution unitarity is lost, since one ends up with a density matrix that is a superposition of density matrices associated with different $t$ 's and that each evolve unitarily according to ordinary quantum mechanics.

Now that we have identified what will play the role of a density matrix in terms of a "real clock" evolution, we would like to see what happens if we assume the "real clock" is behaving semi-classically. To do this we assume that 
$\mathcal{P}_{t}(T)=f\left(T-T_{\max }(t)\right)$, where $f$ is a function that decays very rapidly for values of $T$ far from the maximum of the probability distribution $T_{\max }$. We refer the reader to [6] for a derivation, but the resulting evolution equation for the probabilities is (in the limit in which corrections are small),

$$
\frac{\partial \rho(T)}{\partial T}=i[\rho(T), H]+\sigma(T)[H,[H, \rho(T)]] .
$$

and the extra term is dominated by the rate of change $\sigma(T)$ of the width of the distribution $f\left(t-T_{\max }(t)\right)$.

An equation of a form more general than this has been considered in the context of decoherence due to environmental effects, it is called the Lindblad equation. Our particular form of the equation is such that conserved quantities are automatically preserved by the modified evolution. Other mechanisms of decoherence coming from a different set of effects of quantum gravity have been criticized in the past because they fail to conserve energy [7]. It should be noted that Milburn arrived at a similar equation as ours from different assumptions [8]. Egusquiza, Garay and Raya derived a similar expression from considering imperfections in the clock due to thermal fluctuations [9]. It is to be noted that such effects will occur in addition to the ones we discuss here. Corrections to the Schrödinger equation from quantum gravity have also been considered in the context of WKB analyses [10]. Considering time as a quantum variable has also been discussed by Bonifacio [1] with a formulation somewhat different than ours but with similar conclusions.

Another point to be emphasized, particularly in the context of a volume celebrating Minkowski's contributions, is that our approach has been quite naive in the sense that we have kept the discussion entirely in terms of non-relativistic quantum mechanics with a unique time across space. It is clear that in addition to the decoherence effect we discuss here, there will also be decoherence spatially due to the fact that one cannot have clocks perfectly synchronized across space and also that there will be fundamental uncertainties in the determination of spatial positions. This, together with the issue of the Lorentz invariance of the predictions, is discussed in some detail in our paper [12].

\section{FUNDAMENTAL LIMITS TO REALISTIC CLOCKS}

We have established that when we study quantum mechanics with a physical clock (a clock that includes quantum fluctuations), unitarity is lost, conserved quantities are still preserved, and pure states evolve into mixed states. All this in spite of the fact that the underlying theory is unitary as usual. The effects are more pronounced the worse the clock is. Which raises the question: is there a fundamental limitation to how good a clock can be? This question was first addressed by Salecker and Wigner [1]. Their reasoning went as follows: suppose we want to build the best clock we can. We start by insulating it from any interactions with the environment. An elementary clock can be built by considering a photon bouncing between two mirrors. The clock "ticks" every time the photon strikes one of the mirrors. Such a clock, even completely isolated from any environmental effects, develops errors. The reason for them is that by the time the photon travels between the mirrors, the wavefunctions of the mirrors spread. Therefore the time of arrival of the photon develops an uncertainty. Salecker and Wigner calculated the uncertainty to be $\delta t \sim \sqrt{t / M}$ where $M$ is the mass of the mirrors and $t$ is the time to be measured (we are using units where $\hbar=c=1$ and therefore mass is measured in 1 /second). The longer the time measured the larger the error. The larger the mass of the clock, the smaller the error.

So this tells us that one can build an arbitrarily accurate clock just by increasing its mass. However, Ng and Van Dam 2] pointed out that there is a limit to this. Basically, if one piles up enough mass in a concentrated region of space one ends up with a black hole. Some readers may ponder why do we need to consider a concentrated region of space. The reason is that if we allow the clock to be more massive by making it bigger, it also deteriorates its performance (see the discussion in [13] in response to [14]).

A black hole can be thought of as a clock since it is an oscillator. In fact it is the "fastest" oscillator one can have, and therefore the best clock for a given size. It has normal modes of vibration that have frequencies that are of the order of the light travel time across the Schwarzschild radius of the black hole. (It is amusing to note that for a solar sized black hole the frequency is in the kilohertz range, roughly similar to that of an ordinary bell). The more mass in the black hole, the lower the frequency, and therefore the worse its performance as a clock. This therefore creates a tension with the argument of Salecker and Wigner, which required more mass to increase the accuracy. This indicates that there actually is a "sweet spot" in terms of the mass that minimizes the error. Given a time to be measured, light traveling at that speed determines a distance, and therefore a maximum mass one could fit into a volume determined by that distance before one forms a black hole. That is the optimal mass. Taking this into account one finds that

the best accuracy one can get in a clock is given by $\delta T \sim T_{\text {Planck }}^{2 / 3} T^{1 / 3}$ where $t_{\text {Planck }}=10^{-44} s$ is Planck's time and $T$ is the time interval to be measured. This is an interesting result. On the one hand it is small enough for ordinary times that it will not interfere with most known physics. On the other hand is barely big enough that one might contemplate experimentally testing it, perhaps in future years. 
With this absolute limit on the accuracy of a clock we can quickly work out an expression for the $\sigma(T)$ that we discussed in the previous section [5, 15]. It turns out to be $\sigma(T)=\left(\frac{T_{\text {Planck }}}{T_{\max }-T}\right)^{1 / 3} T_{\text {Planck. With this estimate of the }}$ absolute best accuracy of a clock, we can work out again the evolution of the density matrix for a physical system in the energy eigenbasis. One gets $n$

$$
\rho(T)_{n m}=\rho_{n m}(0) e^{-i \omega_{n m} T} e^{-\omega_{n m}^{2} T_{\text {Planck }}^{4 / 3} T^{2 / 3}} .
$$

So we conclude that any physical system that we study in the lab will suffer loss of quantum coherence at least at the rate given by the formula above. This is a fundamental inescapable limit. A pure state inevitably will become a mixed state due to the impossibility of having a perfect classical clock in nature.

\section{POSSIBLE EXPERIMENTAL IMPLICATIONS}

Given the conclusions of the previous section, one can ask what are the prospects for detecting the fundamental decoherence we propose. At first one would expect them to be dim. It is, like all quantum gravitational effects, an "order Planck" effect. But it should be noted that the factor accompanying the Planck time can be rather large. For instance, if one would like to observe the effect in the lab one would require that the decoherence manifest itself in times of the order of magnitude of hours, perhaps days at best. That requires energy differences of the order of $10^{10} \mathrm{eV}$ in the Bohr frequencies of the system. Such energy differences can only be achieved in "Schrödinger cat" type experiments, but are not outrageously beyond our present capabilities. Among the best candidates today are Bose-Einstein condensates, which can have $10^{6}$ atoms in coherent states. However, it is clear that the technology is still not there to actually detect these effects, although it could be possible in forthcoming years.

A point that could be raised is that atomic clocks currently have an accuracy that is less than a decade of orders of magnitude worse than the absolute limit we derived in the previous section. Couldn't improvements in atomic clock technology actually get better than our supposed absolute limit? This seems unlikely. When one studies in detail the most recent proposals to improve atomic clocks, they require the use of entangled states [16] that have to remain coherent. Our effect would actually prevent the improvement of atomic clocks beyond the absolute limit!

Finally, if one has doubts about the effect's existence, one must recall that one can make it arbitrarily large just by picking a lousy clock. This is of course, not terribly interesting and is not what is normally done in physics labs. But it should be noted that experiments of Rabi oscillations in rubidium atoms measure certain correlations which can be interpreted as having the atom work as a lousy clock. The oscillations show experimentally the exponential decay we discuss. See Bonifacio et al. [17] for a discussion.

\section{CONCEPTUAL IMPLICATIONS}

The fact that pure states evolve naturally into mixed states has conceptual implications in at least three interesting areas of physics. The first two we have discussed before so we only mention them for completeness and to refer the reader to previous papers: the black hole information paradox [5, 15] and quantum computation [18]. The third area where the effects we discussed could be of interest are in foundational issues in quantum mechanics, in particular, the measurement problem. We will now expand a bit on what we mean by this.

The measurement problem in quantum mechanics is related to the fact that in ordinary quantum mechanics the measurement apparatus is assumed to be always in an eigenstate after a measurement has been performed. The usual explanation [19] for this is that there exists interaction with the environment. This selects a preferred basis, i.e., a particular set of quasi-classical states that commute, at least approximately, with the Hamiltonian governing the system-environment interaction. Since the form of the interaction Hamiltonians usually depends on familiar "classical" quantities, the preferred states will typically also correspond to the small set of "classical" properties. Decoherence then quickly damps superpositions between the localized preferred states when only the system is considered. This is taken as an explanation of the appearance to a local observer of a "classical" world of determinate, "objective" (robust) properties.

There are two main problems with such a point of view. The first one is how is one to interpret the local suppression of interference in spite of the fact that the total state describing the system-environment combination retains full coherence. One may raise the question whether retention of the full coherence could ever lead to empirical conflicts with the ascription of definite values to macroscopic systems. The usual point of view is that it would be very difficult to reconstruct the off diagonal elements of the density matrix in practical circumstances. However, at least as a matter of principle, one could indeed reconstruct such terms (the evolution of the whole system remains unitary [20]) 
by "waiting long enough". The second problem is that even if the state ends up being "quasi-diagonal" in the preferred basis of the measurement device, this is not necessarily a completely satisfactory solution to the measurement problem. This is known as the "and-or" problem. As Bell [21] put it "if one were not actually on the lookout for probabilities, ... the obvious interpretation of even $\rho^{\prime}$ [the reduced density matrix] would be that the system is in a state in which various $\mid \Psi_{m}$ >'s coexist:

$$
\left|\Psi_{1}><\Psi_{1}\right| \text { and }\left|\Psi_{2}><\Psi_{2}\right| \text { and } \quad \ldots
$$

This is not at all a probability interpretation, in which the different terms are seen not as coexisting but as alternatives." We will return on this point when we introduce our idea of undecidability later in this paper.

Our mechanism of fundamental decoherence could contribute to the understanding of the two problems mentioned above. In the usual system-environment interaction the off-diagonal terms of the density matrix oscillate as a function of time. Since the environment is usually considered to contain a very large number of degrees of freedom, the common period of oscillation for the off-diagonal terms to recover non-vanishing values is very large, in many cases larger than the life of the universe. This allows to consider the problem solved in practical terms. When one adds in the effect we discussed, since it suppresses exponentially the off-diagonal terms, one never has the possibility that the off-diagonal terms will see their initial values restored, no matter how long one waits.

To analyze the implications of the use of real clocks in the measurement problem, we will discuss in some detail an example. In spite of the universality of the loss of coherence we introduced, it must be studied in specific examples of increasing level of realism. The simplest example we can think of is due to Zurek [22]. This simplified model does not have all the effects of a realistic one, yet it exhibits how the information is transferred from the measuring apparatus to the environment. The model consists of taking a spin one-half system that encodes the information about the microscopic system plus the measuring device. A basis in its two dimensional Hilbert space will be denoted by $\{|+>|-,>\}$. The environment is modeled by a bath of many similar two-state systems called atoms. There are $N$ of them, each denoted by an index $k$ and with associated two dimensional Hilbert space $\left\{\left|+>_{k},\right|->_{k}\right\}$. The dynamics is very simple, when there is no coupling with the environment the two spin states have the same energy, which is taken to be 0 . All the atoms have zero energy as well in the absence of coupling. The whole dynamics is contained in the coupling, given by the following interaction Hamiltonian

$$
H_{\mathrm{int}}=\hbar \sum_{k}\left(g_{k} \sigma_{z} \otimes \sigma_{z}^{k} \otimes \prod_{j \neq k} I_{j}\right) .
$$

In this notation $\sigma_{z}$ is analogous to a Pauli spin matrix. It has eigenvalues +1 for the spin eigenvector $\mid+>$ and -1 for $\mid->$; it acts as the identity operator on all the atoms of the environment. The operators $\sigma_{z}^{k}$ are similar, each acts like a Pauli matrix on the states of the specific atom $k$ and as the identity upon all the other atoms and the spin. $I_{j}$ denotes the identity matrix acting on atom $j$ and $g_{k}$ is the coupling constant that has dimensions of energy and characterizes the coupling energy of one of the spins $k$ with the system. In spite of the abstract character of the model, it can be thought of as providing a sketchy model of a photon propagating in a polarization analyzer.

Starting from a normalized initial state

$$
\mid \Psi(0)>=(a|+>+b|->) \prod_{k=1}^{N} \otimes\left[\alpha_{k}\left|+>_{k}+\beta_{k}\right|->_{k}\right],
$$

it is easy to solve the Schroedinger equation and one gets for the state at the time $t$,

$$
\begin{aligned}
\mid \Psi(t)>= & a \mid+>\prod_{k=1}^{N} \otimes\left[\alpha_{k} \exp \left(i g_{k} t\right)\left|+>_{k}+\beta_{k} \exp \left(-i g_{k} t\right)\right|->\right] \\
& +b \mid->\prod_{k=1}^{N} \otimes\left[\alpha_{k} \exp \left(-i g_{k} t\right)\left|+>_{k}+\beta_{k} \exp \left(i g_{k} t\right)\right|->\right] .
\end{aligned}
$$

Writing the complete density operator $\rho(t)=|\Psi(t)><\Psi(t)|$, one can take its trace over the environment degrees of freedom to get the reduced density operator,

$$
\rho_{c}(t)=|a|^{2}|+><+|+|b|^{2}|-><-|+z(t) a b^{*}|+><-|+z^{*}(t) a^{*} b|-><+|,
$$

where

$$
z(t)=\prod_{k=1}^{N}\left[\cos \left(2 g_{k} t\right)+i\left(\left|\alpha_{k}\right|^{2}-\left|\beta_{k}\right|^{2}\right) \sin \left(2 g_{k} t\right)\right] .
$$


The complex number $z(t)$ controls the value of the non-diagonal elements. If this quantity vanishes the reduced density matrix $\rho_{c}$ would correspond to a totally mixed state ("proper mixture"). That would be the desired result, one would have several classical outcomes with their assigned probabilities. However, although the expression we obtained vanishes quickly assuming the $\alpha$ 's and $\beta$ 's take random values, it behaves like a multiperiodic function, i.e. it is a superposition of a large number of periodic functions with different frequencies. Therefore this function will retake values arbitrarily close to the initial value for sufficiently large times. This implies that the apparent loss of information about the non-diagonal terms reappears if one waits a long enough time. This problem is usually called "recurrence of coherence". The characteristic time for these phenomena is proportional to the factorial of the number of involved frequencies. Although this time is usually large, perhaps exceeding the age of the universe, at least in principle it implies that the measurement process does not correspond to a change from a pure to a mixed state in a fundamental way.

The above derivation was done using ordinary quantum mechanics in which one assumes an ideal clock is used to measure time. If one re-does the derivation using the effective equation we derived for quantum mechanics with real clocks one gets the same expression for $z(t)$ except that it is multiplied by $\prod_{k} \exp \left(-\left(2 g_{k}\right)^{2} T_{\mathrm{Planck}}^{4 / 3} t^{2 / 3}\right)$. That means that asymptotically the off diagonal terms indeed vanish, the function $z(t)$ is not periodic anymore. Although the exponential term decreases slowly with time, the fact that there is a product of them makes the effect quite relevant.

Therefore we see that the inclusion of real clocks makes impossible the observation of "revivals" in systems where the measurement process leads to observable outcomes. The observation of "revivals" not only is very difficult to observe in a practical sense due to the length of time that elapses between revivals, but becomes unobservable due to fundamental reasons, irrespective of the advanced level of the technology used for the observations. As a consequence the revivals are not a means to determine if global coherence in the total system has been preserved or if there has been a change in the state of the measurement system.

Revivals are not the only possible manifestation of the total unitary evolution of the system when an observation of a given result is made in the system. Other experiments have been proposed. These are also of considerable practical difficulty since they involve measurements of the total system. For example, d'Espagnat [23] has proposed a technique based on the observation of constant of the motion of the total system. Such constants of the motion take different values if the reduction process has happened or not. These experiments require the construction of macroscopic ensembles including the environment prepared in the same state and subsequent measurements of each of the degrees of freedom involved. The concrete models proposed [23] for these types of experiments are highly idealized, ignoring interactions between the degrees of freedom of the environment and their individual evolution. The free Hamiltonians of the environment and the measuring apparatus is assumed zero. In these models the loss of unitarity due to real clocks appears to be insufficient to eliminate all possibilities of distinguishing between a reduction and a unitary evolution in an observable process due to the fact that one would be dealing with a constant of the motion. Preliminary analyses of concrete implementations of these ideas in more realistic models lead us to believe that the loss of spatial coherence and dispersion during evolution of the wavefunctions of the component degrees of freedom and the back reaction of the measuring device on the system [24] plus the evolution of the measuring device appear to imply that the observation of the relevant observables is impossible. We are currently studying some of these models and will report elsewhere on the details.

\section{UNDECIDABILITY AND THE LAWS OF PHYSICS}

We have shown that quantum mechanics with and without an explicit mechanism for state reduction yield the same physical predictions. There are good reasons to consider that it is experimentally impossible to distinguished between two alternative situations, the one resulting from a unitary evolution of the system plus the environment and the other one where the system plus the measuring device undergo an abrupt change of state. There are therefore two complete logical structures that yield the same predictions and therefore one cannot decide experimentally which one "corresponds to reality". This is what we refer to as "undecidability".

The concept of undecidability in physics was also discussed by Wolfram [25] in a different context, although not totally disconnected from the one we introduce here. He refers to the undecidability of computational nature about the outcome of a physical process. There exist physical processes whose outcome is not predictable. The optimum computational process to determine the outcome is the physical process itself. In this sense we could speak of undecidability in the outcome of a process. This type of undecidability is weaker than the one we are discussing, since the issues can be decided just by waiting till the outcome occurs. The undecidability we are referring to does not refer to the outcome of a process but to the nature of the process.

In philosophy there are different attitudes that have been taken towards the physical laws of nature (see for instance [26]). One of them is the "regularity theory"; in it, the laws of physics are statements about uniformities 
or regularities of the world and therefore are just "convenient descriptions" of the world. The laws of physics are dictated by a preexisting world and are a representation of our ability to describe the world. Another point of view is the "Necessitarian theory". There laws of nature are "principles" which govern the natural phenomena, that is, the world "obeys" the laws of nature. The presence of the undecidability we point out suggests strongly that the "regularity theory" point of view is more satisfactory.

If one takes seriously the regularity point of view one can ponder about the nature of reality. Does the physical world have a reduction process, does it not, or does it depend on the case? In the case in which there is no reduction process, in the Heisenberg picture the state of a system is given and eternal. If there is a reduction process the state changes every time there is an event resulting in a measurement. The third possibility, which is suggested by the undecidability, is that the system may choose between behaving as if there is a reduction process or not. This would add to the well known probabilistic freedom of quantum systems characterized by the free will theorem of Conway and Kochen [27] another one characterized by two alternative behaviors in relation to the rest of the universe. That is, after the observation of the event either the system simply behaves as if it were part of the universe and its state were that of the universe or if as its state would be given by the reduction postulate. In the first case the system would keep its entanglement with the rest of the universe (i.e. the environment), in the second it will lose its entanglement.

If one adopts what is probably the most attractive view that considers that the universe always evolves unitarily and therefore quantum states are determined once and for all no matter what is the chosen behavior of the subsystem under observation one needs to face the problem of when do events happen in such a framework. Our point of view is that an event occurs when the experimental distinction between coexisting or exclusionary alternatives becomes undecidable, since in that instant the predictions of the laws of physics are not altered by the possible reduction of the state of the system associated with the information acquired when the event takes place.

\section{DISCUSSION}

We have argued that the use of realistic clocks in quantum mechanics implies that pure states evolve into mixed states in spite of the fact that the underlying equations of quantum mechanics are unitary. The effect is further compounded if one allows realistic rulers to measure distances and the formulation can be made Lorentz covariant. This alters fundamentally the picture of space-time as a static fixed entity that was first introduced by Minkowski. Conceptually, it also has profound implications since it allows to construct a quantum mechanics without the introduction of a reduction process that nevertheless has the same predictions as ordinary quantum mechanics with a reduction process. The nature of physical processes is therefore undeterminate in a novel and fundamental way that adds itself to other previous proposals of undecidability in physics.

\section{ACKNOWLEDGEMENTS}

This work was supported in part by grants NSF-PHY0650715, and by funds of the Horace C. Hearne Jr. Institute for Theoretical Physics, FQXi, PEDECIBA (Uruguay) and CCT-LSU.

[1] E. Wigner, Rev. Mod. Phys. 29, 255 (1957).

[2] Y. J. Ng and H. van Dam, Annals N. Y. Acad. Sci. 755, 579 (1995) arXiv:hep-th/9406110; Mod. Phys. Lett. A 9, 335 (1994); see also F. Károlhyázy, A. Frenkel, B. Lukács in "Quantum concepts in space and time" R. Penrose and C. Isham, editors, Oxford University Press, Oxford (1986).

[3] R. Gambini, R. Porto, J. Pullin, Class. Quant. Grav. 21, L51 (2004) arXiv:gr-qc/0305098.

[4] R. Gambini, R. Porto, J. Pullin, New J. Phys. 6, 45 (2004) arXiv:gr-qc/0402118.

[5] R. Gambini, R. Porto, J. Pullin, Braz. J. Phys. 35, 266 (2005) arXiv:gr-qc/0501027.

[6] R. Gambini, R. Porto and J. Pullin, Gen. Rel. Grav. 39, 1143 (2007) arXiv:gr-qc/0603090.

[7] See for instance J. Ellis, J. Hagelin, D.V. Nanopoulos, and M. Srednicki, Nucl. Phys. B241 (1984) 381; T. Banks, M.E. Peskin, and L. Susskind, Nucl. Phys. B244 (1984) 125.

[8] G. J. Milburn, Phys. Rev A44, 5401 (1991).

[9] I. Egusquiza, L. Garay, J. Raya, Phys. Rev. A59, 3236 (1999) arXiv:quant-ph/9811009.

[10] C. Kiefer, T. Singh, Phys. Rev. D44, 1061 (1991).

[11] R. Bonifacio, Nuo. Cim. D114, 473 (1999).

[12] R. Gambini, R. A. Porto and J. Pullin, Int. J. Mod. Phys. D 15, 2181 (2006) arXiv:gr-qc/0611148.

[13] Y. J. Ng and H. van Dam, Class. Quant. Grav. 20, 393 (2003) arXiv:gr-qc/0209021. 
[14] J. C. Baez and S. J. Olson, Class. Quant. Grav. 19, L121 (2002) arXiv:gr-qc/0201030.

[15] R. Gambini, R. A. Porto and J. Pullin, Phys. Rev. Lett. 93, 240401 (2004) arXiv:hep-th/0406260.

[16] See for instance A. Andre, A. Sorensen, M. Lukin, Phys. Rev. Lett 92, 230801 (2004) arXiv:quant-ph/0401130.

[17] R. Bonifacio et al. Phys. Rev. A61, 053802 (2000).

[18] R. Gambini, R. Porto, J. Pullin, in "Gravity, astrophysics and strings at the black sea", P. Fiziev, M. Todorov, editors, St. Kliment Ohridski Press (2006) arXiv:quant-ph/0507262.

[19] M. Schlosshauer, Rev. Mod. Phys. 76, 1267 (2004) arXiv:quant-ph/0312059.

[20] R. Omnès, "The interpretation of quantum mechanics", Princeton Series in Physics, Princeton, NJ (1994).

[21] J. Bell "Against 'Measurement"' in "Sixty two years of uncertainty", A. Miller (editor), Plenum, New York (1990).

[22] W. Zurek, Phys. Rev. D26, 1862 (1982).

[23] B. d'Espagnat, "Veiled reality", Westview, Boulder, Colorado (2003).

[24] S. Bartlett, T. Rudolph, R. Spekkens, P. Turner, New J. Phys. 8, 58 (2006); D. Poulin, J. Yard, New J. Phys. 9 156 (2007).

25] S. Wolfram, Phys. Rev. Lett. 54, 735 (1985).

[26] See for instance "Laws of Nature" in The Stanford encyclopedia of Philosophy (http://plato.stanford.edu) and references therein.

[27] J. Conway, S. Kochen, Found. Phys. 36, 1441 (2006). 